\title{
PROKLA-Redaktion
}

\section{Editorial: China im globalen Kapitalismus}

Vielleicht wird einmal die letzte Februarwoche im Jahr 1972 als ein Wendepunkt in die Geschichtsbücher eingehen, beendete doch der Besuch des damaligen USPräsidenten Richard Nixon bei Mao Zedong in der Verbotenen Stadt die Isolation der Volksrepublik China. Das erklärte Ziel des Treffens, so Nixons Worte, eine Brücke zu bauen, „über die 16.000 Meilen Entfernung und 22 Jahre der Feindschaft, die uns in der Vergangenheit entzweit" hatten, wurde rasch erreicht. Die diplomatischen Kontakte waren in kurzer Zeit wieder hergestellt. Dies ebnete den Weg für den langen Rückmarsch des Reichs der Mitte auf den USamerikanisch dominierten Weltmarkt. Die vorsichtige wirtschaftliche Außenöffnung, die die Kommunistische Partei Chinas schließlich sechs Jahre später einleitete, führte dann dazu, dass das Land seitdem ein stürmisches Wachstum durchläuft. Das rote China hat sich deshalb auch mit einer atemberaubenden Geschwindigkeit dem Kapitalismus zugewandt und gilt heute als eine aufstrebende Weltmacht.

Der Aufstieg Chinas zur zweitgrößten Wirtschaftsnation und zum Exportweltmeister ist mittlerweile auch im Westen unübersehbar geworden. Gerade in der Weltwirtschaftskrise seit 2008 zeigte sich die gewachsene Bedeutung des Landes. China gehörte nicht nur zu den wenigen Ländern weltweit, die nie in eine Rezession geraten waren. Vielmehr hat der chinesische Wachstumsboom maßgeblich dazu beigetragen, dass auch die Konjunktur in anderen Weltregionen wieder angesprungen ist. Doch die Faszination über diese rasante Entwicklung ist bei manch einem Kommentator der Angst gewichen. Die panische Feststellung des Hochglanzmagazins Fortune, dass China dabei sei, „die Welt zu kaufen“, drückt einen allgemeinen Stimmungswandel aus. China hat aufgrund seiner gewaltigen Warenausfuhren heute mit rund 2,6 Billionen US\$ - das entspricht ungefähr dem Bruttoinlandsprodukt Frankreichs - riesige Devisenreserven angehäuft, die jederzeit dazu genutzt werden könnten, im Ausland zu investieren. Spektakuläre Übernahmen von Traditionsunternehmen im Norden durch kapitalstarke chinesische Konzerne - etwa der Kauf der PC-Sparte von IBM durch den Computerhersteller Lenovo oder die Übernahme von Volvo durch den Shanghaier Autohersteller Geeley - sind zwar noch nicht zur Regel geworden, werden zumindest aber argwöhnisch beäugt. Selbst im Finanzsektor, lange Zeit eine unbestrittene Domäne US-amerikanischer, europäischer und japanischer Finanzinstitute, ist ein Umbruch zu beobachten. Die Top Ten der weltweit größten Banken werden heute gleich von drei chinesischen Instituten angeführt. Die jüngsten Zeitungsmeldungen, dass der politisch geschwächte US-Präsident Barack Obama nun den ersten Rang im Forbes-Ranking der mächtigsten Menschen der Welt an Hu Jintao, dem Generalsekretär der KPCh und Staatspräsidenten Chinas, abtreten musste, erscheinen somit als logische Konsequenz dieser Geschehnisse. 
Folglich ist die ökonomische Machtverschiebung nicht ohne politische Folgen geblieben. Die Zweckehe zwischen den USA und China, die einst zwischen Mao und Nixon geschlossen wurde und sich damals gegen die Sowjetunion richtete, ist mittlerweile in die Tage geraten. Auf dem G-20-Gipfel im November 2010 in Seoul herrschte eine eisige Stimmung zwischen den Partnern. Immer lauter wurden die Forderungen der USA, dass China seine Währung aufwerten und seine massiven Leistungsbilanzüberschüsse abbauen möge, während der jüngste Versuch der US-Notenbank, dem notorischen Schuldnerland durch den erneuten Kauf von Staatsanleihen frisches Geld zuzuführen, von den chinesischen Behörden mit Besorgnis kommentiert wurde. Dass ausgerechnet die staatliche chinesische Ratingagentur Dagong Global Credit Rating dies zum Anlass nahm, die Kreditwürdigkeit der USA herabzustufen, zeigt deutlich, dass in China ein Konsens herrscht, sich nicht mehr freiwillig den Vorgaben aus Washington unterzuordnen. Die Verschlechterung der Wetterlage im US-amerikanisch-chinesischen Verhältnis, das zeitweilig sogar unter dem Begriff „Chimerica“ als ein symbiotisches beschrieben wurde, deutet darauf hin, dass nicht nur die bilateralen Beziehungen beider Länder, sondern mittelfristig sogar das gesamte Regelwerk der Weltwirtschaft auf dem Spiel stehen könnte, sind China und die USA doch die bedeutendsten Volkswirtschaften der Welt. Eine solche Transformation wird freilich nicht harmonisch verlaufen, da - dies lehrt die Erfahrung früherer Aufstiegsprozesse - die etablierten Mächte im Weltsystem ihren Einfluss über Rohstoffe und Märkte nicht einfach kampflos aufgeben werden.

$\mathrm{Ob}$ und wie der Aufstieg Chinas weiter verläuft und welche Konflikte er hervorruft, wird in den Beiträgen dieses Heftes untersucht. Einen Überblick über die Debatte zum Aufstieg Chinas gibt Stefan Schmalz. Er identifiziert mit den Weltsystemansätzen als Vertreter der These eines Aufstiegs Chinas und den kritischen Ansätzen der Internationalen Politischen Ökonomie als Verfechtern einer Kontinuität der US-Hegemonie zwei Strömungen, die unterschiedliche Deutungen anbieten. Schmalz überprüft deren Argumente anhand von Grunddaten zur Entwicklung des chinesischen Wirtschaftsmodells sowie zur Macht Chinas in der Weltwirtschaft und fragt, welche Konflikte Chinas Aufstieg erzeugen wird. Im ersten Teil des Heftes werden verschiedene Interpretationen des chinesischen Aufstiegs diskutiert. Ingo Schmidt schließt sich in seinem Beitrag der aufstiegskritischen Sichtweise an. Er vertritt die These, dass China ähnlich wie Deutschland und Japan durch sein exportgeleitetes Wirtschaftsmodell nicht in der Lage ist, die US-amerikanische $\mathrm{He}$ gemonie herauszufordern. Vielmehr ist Chinas ökonomische Entwicklung stark außenabhängig, sodass die großen Konjunkturprogramme zur Förderung der chinesischen Wirtschaft in der Krise 2008 auch zu keiner Veränderung des Wachstumsmodells geführt haben. Gerhard Armanski geht hingegen von einer globalhistorischen Perspektive aus. Er stellt die Gründe für den Niedergang Chinas im 19. Jahrhundert dar, nachdem das Land bis zu diesem Zeitpunkt den europäischen Staaten in jeder Hinsicht technologisch, wirtschaftlich und militärisch - das Wasser reichen konnte. Armanski sieht die Gründe hierfür sowohl in internen Entwicklungsblockaden und Konflikten als auch im aggressiven Vordringen des westlichen und später des japanischen Imperialismus nach dem Opium-Krieg 1840-42. Das historische Verdienst der $\mathrm{KPCh}$ besteht deswegen vor allem darin, China von der Dominanz 
des Westens befreit zu haben. Ngai-Ling Sum widmet sich in ihrem Beitrag den Diskursen um den Aufstieg der BRICStaaten in der Weltwirtschaft. Dafür nutzt sie den Ansatz der Kulturellen Politischen Ökonomie, um die Strategien und Interessen von transnationalen Akteuren wie Investmentbanken herauszuarbeiten, die diese Diskurse eingeleitet haben. Sie identifiziert einen Formwandel der diskursiven Strategie und kommt zum Schluss, dass die Aufwertung der BRIC-Staaten zur Stabilisierung des globalen Neoliberalismus genutzt wird.

Im zweiten Teil des Heftes werden die regionalen Auswirkungen des chinesischen Aufstiegs untersucht. Tobias ten Brink rückt in seiner Analyse Ostasien in den Mittelpunkt. Er untersucht die Besonderheiten des ostasiatischen Verflechtungsraums als ein wirtschaftlich stark integriertes, aber nur locker institutionalisiertes Gebilde und beobachtet Chinas wachsende Rolle im ostasiatischen Kontext. Dabei sieht ten Brink die Tendenz, dass China zunehmend die USA als Hauptbezugspunkt für politische und wirtschaftliche Kooperation ersetzt. Dieser Prozess zeigt, dass die regionale Ordnung in Ostasien eine äußerst instabile Konfiguration ist und zu einem Kampffeld der USA und China werden könnte. Jörg Goldberg untersucht die wachsende Rolle Chinas (und auch Indiens) auf dem afrikanischen Kontinent. Er sieht zwar ähnliche Handelsbeziehungen zwischen China als Exporteur von Industriewaren und Afrika als Rohstoffproduzenten im Entstehen begriffen, wie sie bereits zwischen dem Westen und Afrika gängig sind. Allerdings stellt China keine politischen Vorgaben für Wirtschaftsverträge und Afrika hat zudem durch China als Alternative massiv an Verhandlungsmacht gewonnen. Er kommt deswegen zum Schluss, dass es derzeit vor allem in den Händen der afrikanischen Politik liegt, die neuen Chancen zu nutzen.

Zuletzt werden die sozialen Konflikte analysiert, die in China entstanden sind. Beverly Silver und Lu Zhang vertreten die These, dass „wo das Kapital hingeht, der Konflikt zwischen Arbeiterklasse und Kapital bald nachfolgt" und sehen deshalb China als neuen Mittelpunkt der globalen „Arbeiterunruhe“. Die neuen Protestaktivitäten könnten zu einer grundlegenden Veränderung der industriellen Beziehungen Chinas führen. Das chinesische Arbeitsvertragsgesetz von 2008 wird von den beiden Autorinnen mit dem 1935 in den USA verabschiedeten National Labor Relations Act verglichen. Dieser trug dazu bei, dass damals eine Streikwelle in den USA entstand, die Roosevelts New Deal-Politik begünstigte. Die derzeitigen Arbeitskämpfe in China erlangen aus diesem Blickwinkel eine globalhistorische Bedeutung. Florian Butollo untersucht vor diesem Hintergrund die Streikwelle im Sommer 2010 in China. Er arbeitet die Besonderheiten der industriellen Beziehungen in China und der Arbeitsniederlegungen heraus: Zwar haben diese durch die offensiven Lohnforderungen, die basisdemokratischen Elemente und die innerbetrieblichen Konflikten mit den früheren Mustern der eher eruptiven, unorganisierten Proteste gebrochen. Aber aufgrund der unterschiedlichen Ausgangsbedingungen, etwa der zerklüfteten industriellen Landschaft, kann bisher nicht von einer einheitlichen Arbeiterbewegung gesprochen werden. Butollo stimmt Silver und Zhang deswegen nur teilweise zu: Wo das Kapital hingeht, treten zwar Konflikte auf - nur eben nicht gleichzeitig, nicht am selben Ort und auch nicht unter ähnlichen Interessenkonstellationen. 


\section{Durchsuchungsaktionen beim linken Buchhandel}

Im Sommer kam es zu mehreren von der Staatsanwaltschaft veranlassten polizeilichen Hausdurchsuchungen von linken Buchhandlungen. Die Redaktion der PROKLA erklärt sich mit den betroffenen Buchhandlungen solidarisch und verurteilt die polizeiliche Vorgehensweise. Buchhandlungen sind ein wichtiges Organ der aufgeklärten und öffentlichen Meinungsbildung, denn von der Existenz vieler Publikationen kann die Öffentlichkeit nur dadurch erfahren, dass sie in Buchhandlungen zu finden sind. Aus einer Reihe von Gründen ist die Situation des Buchhandels insgesamt, also sowohl der Verlage als auch der Buchläden, prekär. Die Gefahr ist nicht gering, dass diese Situation insbesondere für den linken Buchhandel durch polizeiliche Untersuchungen noch weiter verstärkt wird. Denn sie können dazu beitragen, dass Buchhandlungen ihr politisches Ziel, nämlich auch kritische Literatur interessierten Kunden anzubieten, nicht mehr wahrnehmen können. Dies gilt nicht nur aus ökonomischen Gründen, weil möglicherweise ein Teil der Kundschaft abgeschreckt wird, sondern mehr noch aus politischen Gründen selbst. Denn offensichtlich wird von staatlicher Seite von den Buchhändlern erwartet, dass sie die von ihnen zum Verkauf angebotenen Schriften daraufhin prüfen, ob sie formal oder inhaltlich geltendem Recht entsprechen. Das kann nicht Aufgabe des Buchhandels sein, dass er in die Rolle eines Zensors gedrängt wird. Als Redaktion einer gesellschaftskritischen Zeitschrift haben wir ein kollegiales Verhältnis zum linken Buchhandel, dennoch hätten wir Bedenken, wenn wir befürchten müssten, dass Analysen und Argumente nicht in der kritischen Öffentlichkeit diskutiert würden, sondern der Buchhandel dahin gedrängt würde, durch Sortimentspolitik das Spektrum der Meinungsbildung zu verengen.

$$
* * *
$$

Eine traurige Nachricht zum Schluss. Im September ist unser Autor und früheres Redaktionsmitglied Michael Stanger verstorben. Michael war von 1984-1986 Mitglied der Redaktion. Er hat am Otto Suhr Institut der FU Berlin Politikwissenschaft studiert, seine (immer noch lesenswerte) Dissertation „Krisentendenzen der Kapitalakkumulation. Theoretische Kontroversen und empirische Befunde" erschien 1988. Michael wurde 57 Jahre alt. 